\title{
Who's Who with Big-Five: Analyzing and Classifying Personality Traits with Smartphones
}

\author{
Gokul Chittaranjan ${ }^{1,2}$, Jan Blom ${ }^{3}$, Daniel Gatica-Perez ${ }^{1,2}$ \\ gthatta@idiap.ch, jan.blom@nokia.com, gatica@idiap.ch \\ ${ }^{1}$ Idiap Research Institute, Centre du Parc, PO Box 592, Rue Marconi 19, 1920 Martigny, Switzerland \\ ${ }^{2}$ Ecole Polytechnique Federale de Lausanne, 1015 Lausanne, Switzerland \\ ${ }^{3}$ Nokia Research Center Lausanne, PSE-C, EPFL, 1015 Lausanne, Switzerland
}

\begin{abstract}
In this paper, we investigate the relationship between behavioral characteristics derived from rich smartphone data and self-reported personality traits. Our data stems from smartphones of a set of 83 individuals collected over a continuous period of 8 months. From the analysis, we show that aggregated features obtained from smartphone usage data can be indicators of the Big-Five personality traits. Additionally, we develop an automatic method to infer the personality type of a user based on cellphone usage using supervised learning. We show that our method performs significantly above chance and up to $75.9 \%$ accuracy. To our knowledge, this constitutes the first study on the analysis and classification of personality traits using smartphone data.
\end{abstract}

\section{Introduction}

The rapid global growth of mobile phone usage [1] has reinforced the need to study the psychological, social, and economic implications of mobile telephony. Smartphones provide a new lens for investigating mobile phone usage [22]. Such platforms are programmable, allowing the development of data collection tools to record various behavioral aspects of the user, ranging from how the device is used across different contexts to analyzing spatial and social dimensions of the everyday life of the user, as captured through sources like GPS, call logs, and Bluetooth. From the point of view of designing communication features and applications that are tailored to the individual needs and preferences of a user, this data intensive framework provides a wealth of new opportunities, as it allows us to understand the impact of context on user behavior as well as to study individual differences, such as personality of the users.

In personality psychology, personality traits play a central role in describing a person [17]. This topic has also been found to be of vital importance in computing. Several studies have been recently conducted on personality traits and their relationship to the use of Internet and forms of social media such as Youtube, blogs, Facebook and other social networks [3, 6, 24, 26].

Since mobile phones also mediate social interactions, phone usage could reflect an individual's personality [5]. However, in contrast to the significant amount of research in the web context, surprisingly few studies have been carried out in the past to investigate the connection between mobile phone usage and personality of individuals. In particular, the following points have not yet been adequately addressed: First, there is a clear need for scalability of studies to both a large and diverse feature set and a user base. This has not been possible in the past because of the burden on a user, who is often a customer, in answering lengthy questionnaires. Second, the rich contextual information that can be extracted with current smartphones has not been studied from the perspective of personality. Third, the automatic inference of usage or traits, based on features that can be reliably extracted from continuously collected data has not been explored.

Determining the personality of mobile phone users, besides being important solely from the psychological point of view, can also provide an interesting framework for wearable computing. The ability to draw connections between behavioral aspects derived through contextual data collected by mobile phones, as well as personality, could lead to designing and applying machine learning methods to classify users into personality types. Such understanding could be used in various ways. For instance, prior research has shown that personality is linked to user interface preferences, 
like the surface color of an application [4]. Certain personality traits, like extraversion/introversion, have also been found to be linked to preferences pertaining to visual aesthetics of web sites [11]. The personality of a user might also determine the kind of functionality the individual is disposed to use on the phone. Individual differences in personality may also correlate with the impact of context on the user. For instance, when faced with idle time, is an extraverted person likely to use the device in a different way, as compared to an introvert? The preferred interaction modalities may also differ across personality types. Conscientious persons, for example, may be prone to switch their devices to a silent mode in a socially sensitive situation.

Although the examples given above are hypothetical, they nevertheless indicate that expending efforts on establishing a link between personality and behavior can be justified by the wealth of design opportunities such a discovery would lead to. In this work, we analyze the relationship between smartphone usage and the personality traits in the Big-Five Model [17], and develop an automatic method to classify users according to self-perceived personality using features that are by nature privacy sensitive and extracted from usage logs and phone sensors on the Nokia N95 smartphone. Our experiments are based on subset of the Lausanne Data Collection Campaign[12], and contains data continuously collected from 83 participants over 8 months of time. First, we show that significant relationships exist between personality traits and some of the extracted features using correlation and multiple regression analyses. Next, we describe an automated method to classify users according to their personality traits. We show that our method's performance is promising. To our knowledge, this constitutes the first study on inferring personality traits of smartphone users from automatically extracted features.

The paper is organized as follows. Section 2 describes past work relating to personality measurement through direct or indirect means. The dataset used along with details about feature extraction is given in section 3. The correlation analysis between features and personality, and subsequently the classification of users based on their Big-Five traits is described in section 4. Finally, we conclude in section 5.

\section{Related Work}

The Big-Five personality framework [17] has received considerable support in psychology in the past, although there has not been a universal acceptance of the concept. This framework is a hierarchical model of personality traits that represent personality at the broadest level of abstraction [10]. It consists of five bipolar factors, namely extraversion, agreeableness, conscientiousness, neuroticism, and openness to experience [17]. These factors, described in Table 1, summarize several more specific traits and are believed to capture most of the individual differences in human personality [10].

Given the objectives of this work, it is useful to contrast personality assessment methods into questionnaire and behavior based. The questionnaires used in many Big-Five personality studies are typically lengthy. This can be a limitation when a large number of participants at geographically spread areas have to complete questionnaires online. Therefore, efforts have been made to develop brief scales in psychology [10], so as to minimize the time required by the participants to fill in a survey as well as the cost associated with the process of filling in questionnaires. In this context, Gosling et. al. introduced the Ten Item Personality Inventory (TIPI) [10] that includes, as the name suggests, ten questions to determine the Big-Five personality traits. It has been shown that the TIPI instrument reaches adequate convergence with the Big-Five measures in self-reported ratings [10]. Hence, in our study we use TIPI to measure self-perceived personality.

On the other hand, in relation to assessing personality indirectly, through behavioral characteristics Pianesi et. al. showed that personality traits in a meeting environment can be detected using audio-visual features and supervised learning [19]. In this case, personality of the participants was revealed by how participants spoke and interacted in the experimental situation. Similarly, Mairesse and Walker describe an automatic procedure using NLP and audio features to detect the Big-Five traits from conversation extracts $[15,16]$. While the above examples highlight that behavior can be indicative of the personality of an individual, the role of the mobile phone in revealing this behavior remains a relatively unexplored territory. This is surprising given that there is plenty of prior research pertaining to modeling users and their mobile phone usage patterns. To name a few examples, Eagle and Pentland described the concept of eigenbehavior and its usefulness in predicting behavioral patterns and ties in a network of people [8]. Farrahi and Gatica-Perez have illustrated ways of determining routines of users by modeling sensor data pertaining to location collected from mobile phones using topic models [9]. Further, Do and Gatica-Perez [7] recently presented an analysis of application usage in smartphones, for the purpose of user retrieval. Similarly, Verkasalo et. al. studied the reasons and motivation behind using applications across users and non-users [25]. These studies tie well with the thriving "app-usage" culture established by smartphone manufacturers - through services like the 
Table 1: Big-Five traits and examples of adjectives [17]

\begin{tabular}{ll}
\hline Trait & Examples of Adjectives \\
\hline Extraversion (E) & Active, Assertive, Energetic, Enthusiastic, Outgoing, Talkative \\
Agreeableness (A) & Appreciative, Forgiving, Generous, Kind, Sympethetic \\
Conscientiousness (C) & Efficient, Organized, Planful, Reliable, Responsible, Thorough \\
Neuroticism (N) & Anxious, Self-pitying, Tense, Touchy, Unstable, Worrying \\
Openness to Experience (O) & Artistic, Curious, Imaginative, Insightful, Original, Wide Interests \\
\hline
\end{tabular}

Apple App Store, Nokia Ovi Store and the Android Market. However, very few studies have directly addressed the relationship between smartphone usage and personality, although personality plays a vital role in social science and psychology.

In a related study, Poschl and Doring presented an analysis relating usage patterns in phones to users clustered on the basis of big-five personality traits into two discrete groups. All information in the study was gathered using questionnaires [20]. Recently, Butt and Phillips presented a study of personality and its relationship to mobile phone usage [5]. The detailed NEOFFI personality test in conjunction with the Coopersmith self-esteem inventory were administered to participants of the study. Factors describing levels of phone usage were obtained from another questionnaire. The features used in this study were related to phone calls and SMS usage. Many of the comparisons made in the study were motivated by previous work investigating the link between personality traits and Internet usage [5]. In this study, disagreeable individuals tended to be more likely to report receiving more calls and also a higher proportion of calls as "unwanted". Outgoing calls were not significantly explained by the traits. Extraverted, neurotic, and non-conscientious individuals were reported to have spent more time sending/receiving SMS, and extraverted and disagreeable individuals were found to spend more time changing the ring tone or wallpapers. In a similar work, Phillips et. al. [18] also found that disagreeable individuals were more likely to play games on their phone [18].

Our study differs from past work in several ways. We utilize information available in today's smartphones, such as the usage of web, music, video, maps, proximity information derived from bluetooth etc., in addition to the traditional call and SMS usage information. All cues are automatically extracted from usage logs, without intervention or input from users. Therefore, we do not rely on personal recall of these usage cues, that can be prone to human errors and biases. We use a short personality questionnaire that makes the project scalable to a large population. Finally, we also device an automatic method, using supervised learning to classify users according to the Big-Five traits.

\section{$3 \quad$ Dataset and Feature Extraction}

In our work, we use smartphone data of 83 participants who participated in the Lausanne data collection campaign [12], a people sensing project organized in French-speaking region of Switzerland. We use data collected for a continuous period of 8 months (between November 2009 and July 2010) using a continuous nonintrusive, passive data collection software running on Nokia N95 phones. This software collected anonymized logs of calls (Call Logs), SMS (SMS Logs), bluetooth scans (BT Logs), and application usage (App Logs).

Of the 83 participants, 53 were male and 28 were female, 2 participants chose not to disclose their gender. The mean age was 29.7 years with a standard deviation of 7.6 years. The minimum and maximum ages were 19 and 63 years respectively. 63 participants had at least a university degree. Among the participants, 38 were Asians, 2 were North Americans and 42 were Europeans. All users were previous mobile phone users, but most of them had not owned a smartphone before the study. Therefore, they discovered most of the features of the N95 phone during the study.

Self-perceived personality was measured using the TIPI questionnaire [10]. This was obtained as a part of an online exit survey administered in two languages, based on the language preference. The TIPI questionnaire could be answered within a few minutes.

The features used in our studies are aggregated from the logs on a monthly level. In other words, all users were split across months, which gave us 567 usermonths. From each of the user-months, features describing different aspects of a smartphone usage were computed automatically, by parsing the logs, as summarized in Table 2. The selection of features is based on previous work enlisted in section 2 and on the choice of features that could reasonably characterize levels of individual and relational activity. All features except those from BT Logs were obtained by aggregating events (such as the opening of an Office or Internet application) as and when they happened. Features pertaining to Bluetooth, were based on scans done approximately once every 3 minutes. Defining a time slot as one bluetooth scan, we also computed features that captured the duration for which an ID was available. 
Table 2: Usage cues extracted from smartphone data aggregated on a monthly basis

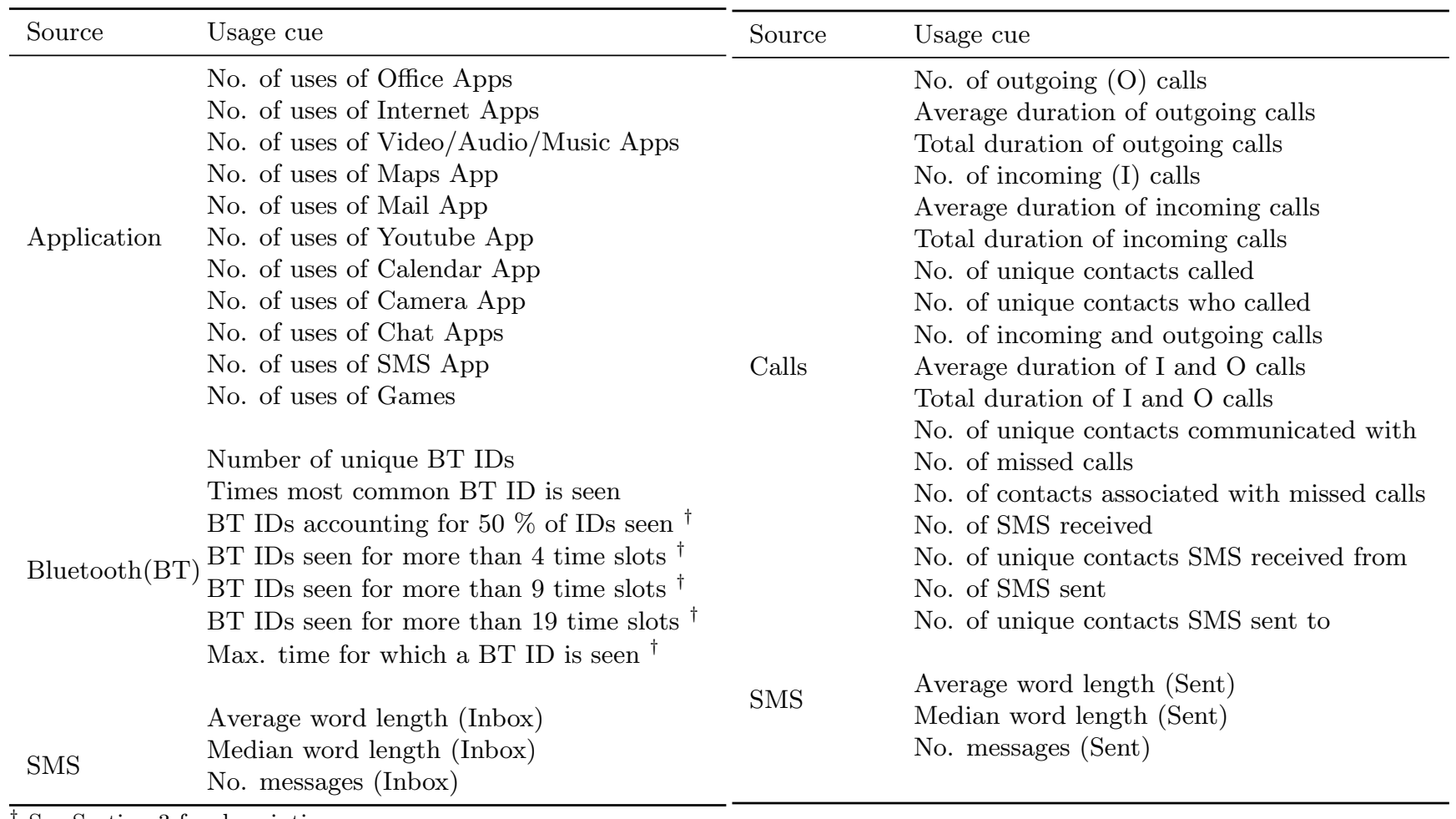

$\dagger$ See Section 3 for description

\section{Experiments and Results}

We systematically analyze the relationship between personality traits and usage features in the following section, using standard correlation and multiple regression analyses. Next, we present a machine learning approach to classify users according to their big-five traits using SVM and C4.5 classifiers.

\subsection{Data Analysis}

\subsubsection{Analysis of independent variables}

The mean and standard deviation for the five traits, along with the minimum and maximum value and skewness is tabulated in Table 3. It should be noted that Neuroticism is replaced by Emotional Stability, since this was computed from the TIPI questionnaire. None of the predictors had a skew $\geq 1$ or $\leq-1$, hence no transformation was applied to the data.

The correlations between the traits are given in Table 4. Although significant correlations (as high as 0.64 between agreeableness and emotional stability) exist, all were below the selection criteria used in the test for multi-collinearity in previous work [5].

\subsubsection{Analysis of Dependent Variables}

All the automatically extracted features were found to be positively skewed, so a log-transformed transformation, $\log ($ feature +1$)$ was applied for the correlation and multiple regression analyses. We first found that the features derived from App Logs were very sparse, due to the low usage frequency of some applications. Hence, for analyses involving App Logs, we chose only those user-months for which there has been some use of a given application. Further, for all analyses, only those user-months with at least 7 days of usage of either calls, SMS, BT or applications were chosen. This was done to avoid user months that might contain no data, owing to a variety of reasons such as vacations, problems with phone use etc.

Pearson's correlation coefficient between the features and the traits, as well as multiple regression analyses between each of the features against all the independent variables (traits) were done. These results are tabulated in Tables 5 and 6 respectively. The standardized regression coefficient $(\beta)$ and $t$-statistics were also computed. For purposes of clarity, the discussion to follow has been organized based on the source of features. App Logs: It was found that the usage of all applications, except the use of Maps, Camera, Chat and Game applications significantly explained variance in the traits. Multiple regression analysis showed that the traits accounted for $12 \%$ of the variance in the use of Office, with conscientious $(\beta=0.25, t=3.01, p=$ $0.003)$, not emotionally stable (neurotic) $(\beta=-0.22$, $t=-2.29, p=0.023)$ and low-openness $(\beta=-0.29$, $t=-3.97, p<0.001)$ participants to use it more. 
Table 3: Statistics for the Big-Five traits

\begin{tabular}{llllll}
\hline Predictors & Mean & SD & Min. & Max. & Skew \\
\hline Extraversion & 4.09 & 1.24 & 1.00 & 7.00 & -0.18 \\
Agreeableness & 4.88 & 1.28 & 2.00 & 7.00 & -0.39 \\
Conscientiousness & 5.10 & 1.42 & 1.00 & 7.00 & -0.71 \\
Emotional Stability & 4.61 & 1.24 & 1.50 & 7.00 & -0.28 \\
Openness to Experience & 4.69 & 1.42 & 1.00 & 7.00 & -0.64 \\
\hline
\end{tabular}

Table 4: Correlations across the 5-independent variables (Big-Five Traits)

\begin{tabular}{|c|c|c|c|c|c|}
\hline Predictors & 1 & 2 & 3 & 4 & 5 \\
\hline 1. Extraversion & & -0.06 & -0.07 & $-0.29^{* *}$ & 0.11 \\
\hline 2. Agreeableness & & & $0.50^{* *}$ & $0.64^{* *}$ & $0.41^{* *}$ \\
\hline 3. Conscientiousness & & & & $0.50^{* *}$ & $0.50^{* *}$ \\
\hline 4. Emotional Stability & & & & & $0.39^{* *}$ \\
\hline 5. Openness to Experience & & & & & \\
\hline
\end{tabular}

Further, we found that the Mail application was more likely to be used by neurotic $(\beta=-0.27, t=-2.32$, $p=0.021)$ and conscientious $(\beta=0.33, t=2.92$, $p=0.004)$ participants. This trend is similar to that observed with the use of office applications. A potential explanation to this correlation is that these applications are used with relation to work. Hence participants using e-mail to communicate might be expected to be more responsible and efficient. Interestingly, introverts $(\beta=-0.2575, t=-5.246, p<0.001)$ were less likely to use Internet applications on the phone. This is also reinforced by the significant negative pairwise correlation observed in Table $5(r=-0.26, p<$ 0.01). Although the use of Audio/Video/Music applications was explained only to an extent of $4 \%$ by the traits, upon examining the regression coefficients, we observed that conscientious individuals were less likely to use them $(\beta=-0.21, t=-3.735, p<0.001)$. This relationship also shows up in the form of a significant negative correlation in Table $5(r=-0.18, p<0.01)$.

Finally, the SMS application (which might not correspond to the actual number of SMS sent/received) was found to be more likely to be used by disagreeable $(\beta=-0.15, t=-2.61, p=0.01)$, conscientious participants $(\beta=0.16, t=3.07, p=0.002)$ who score low on openness $(\beta=-0.24, t=-4.74, p<0.001)$. Conscientious individuals are planful, thorough and responsible, while disagreeableness relates to being unforgiving and showing low trust. These factors could have contributed to the SMS applications usage trends.

BT Logs: None of the BT features explained a large percentage of variance in the traits. We observed that introverts $(\beta=-0.12, t=-2.60, p=0.010)$ and disagreeable $(\beta=-0.15, t=-2.48, p=0.01)$ participants had fewer number of unique BT IDs scanned. This could be because of introverts and disagreeable individuals being less social in nature. The number of BT IDs seen multiple times (4, 9, and 19) showed a consistent trend with positive $\beta$ values for emotional stability and negative $\beta$ values for agreeableness, suggesting that emotionally stable and disagreeable individuals might encounter BT IDs for longer durations. This might be due to emotionally stable and disagreeable participants having a tendency of staying in the same place for longer periods of time.

SMS Logs: Multiple regression analysis on our data showed that message size in the inbox were not significantly explained by the traits. However, the number of messages in the inbox showed that emotionally stable users $(\beta=0.18, t=2.86, p=0.004)$ scoring low on openness $(\beta=-0.15, t=-3.00, p=0.002)$ were more likely to have more messages. Features describing the quality of messages in the sent folder had their variance explained significantly by the traits. Emotionally stable individuals (For Avg. word length feature, $\beta=0.16, t=2.61, p=0.009)$ with lowopenness (For Avg. word length feature, $\beta=-0.19$, $t=-3.77, p<0.001)$ were found to use longer words. They also had more items in the sent folder. This suggests that the personality of a user may be better understood using SMS composed by him, rather than the messages he receives.

Call Logs: While the number and duration of outgoing calls was not significantly explained by the five traits, the variance in the number and duration of incoming calls and the number of unique contacts associated with them, were significantly explained. Extraverts $(\beta=0.18, t=3.98, p<0.001)$ and agreeable $(\beta=0.15, t=2.59, p=0.01)$ individuals were likely to receive more calls. This is also seen in pairwise correlations with $r=0.13$ and 0.20 for extraversion and agreeableness respectively. The duration of 
Table 5: Pairwise correlations between features and traits having $p<0.01$, ranked by absolute value of $r$

\begin{tabular}{|c|c|c|c|}
\hline Feature & $\mathbf{r}$ & Feature & $\mathbf{r}$ \\
\hline \multicolumn{2}{|l|}{ Extraversion } & \multicolumn{2}{|l|}{ Conscientiousness } \\
\hline Uses of Internet & -0.26 & Uses of Video/Audio/Music & -0.18 \\
\hline Total duration of incoming calls & 0.20 & No. BT IDs accounting for $50 \%$ of IDs seen & -0.14 \\
\hline Average duration of incoming calls & 0.18 & Times most common BT ID is seen & 0.14 \\
\hline Uses of Camera & -0.15 & Unique contacts SMS sent to & -0.13 \\
\hline $\begin{array}{l}\text { Avg. word length (sent) } \\
\text { Median word length (sent) }\end{array}$ & $\begin{array}{l}-0.15 \\
-0.15\end{array}$ & \multicolumn{2}{|l|}{ Emotional Stability } \\
\hline Calls received & 0.13 & Uses of Office & -0.23 \\
\hline SMS sent & -0.13 & Unique contacts that called & 0.16 \\
\hline No. unique BT IDs & -0.13 & Uses of calendar & -0.16 \\
\hline \multicolumn{2}{|l|}{ Agreeableness } & Calls received & 0.15 \\
\hline \multirow{10}{*}{$\begin{array}{l}\text { Incoming calls } \\
\text { Uses of office } \\
\text { Uses of Calendar } \\
\text { Unique contacts called } \\
\text { Total duration incoming calls } \\
\text { Unique contacts SMS sent to } \\
\text { BT IDs seen more than } 4 \text { times } \\
\text { BT IDs accounting for } 50 \% \text { of IDs seen }\end{array}$} & \multirow{10}{*}{$\begin{array}{l}0.20 \\
-0.18 \\
-0.18 \\
0.17 \\
0.13 \\
-0.13 \\
-0.12 \\
-0.11\end{array}$} & Median word length (sent) & 0.14 \\
\hline & & \multicolumn{2}{|l|}{ Openness to Experience } \\
\hline & & Uses of Office & -0.26 \\
\hline & & Uses of Calendar & -0.18 \\
\hline & & No. messages (sent) & -0.18 \\
\hline & & Uses of SMS & -0.17 \\
\hline & & Uses of Internet & -0.15 \\
\hline & & Total duration of incoming calls & 0.13 \\
\hline & & Avg. duration of incoming calls & 0.12 \\
\hline & & Missed calls & -0.12 \\
\hline
\end{tabular}

calls was also more likely to be higher for extraverts, both in average and total (for average duration of calls, $\beta=0.18, t=3.81, p<0.001$, refer Table 5 for $r$ values). Reinforcing these results, we found that the number of unique contacts who called was likely to be higher for extraverts $(\beta=0.13, t=2.74, p=0.006)$. Finally, regression coefficients showed that individuals scoring higher on openness were less likely to miss calls $(\beta=-0.18, t=-3.62, p<0.001)$. They were also less likely to send SMS. This concurs with the results obtained from SMS Logs.

Comparison to previous studies: To our knowledge, this is the first study utilizing automatically extracted cues from multiple sources to analyze personality. Past work $[5,18]$ has explored self-reported usage of phones for calls, SMS and games based on surveys and personal recollection. Interestingly, past results show that extraverted and disagreeable participants reported more incoming calls [5], while our study suggests that extraverted and agreeable individuals were more likely to receive incoming calls. In our study, no significant $t$-values were found for the duration spent in receiving calls and agreeableness but it was found that extraverts spent more time on incoming calls. Additionally, our results show that the time spent in incoming and outgoing calls did not significantly explain the variance in the traits and a slightly positive $\beta$ (not sig- nificant) was observed for both extraversion and agreeableness. This contrasts with the previous study [5], in which extraverted and disagreeable individuals were more likely to be found spending time making and receiving calls. These observations could be attributed to two reasons. Self-perception of usage levels among disagreeable individuals might be different from that of actual usage. Further, agreeableness was found significantly positively correlated with conscientiousness. This could have indirectly contributed to the difference. Finally, outgoing calls were not significantly explained by the traits in the present and previous study [5], suggesting that it is a less-discriminative feature.

Disagreeableness was a predictor for SMS app usage in our study. This concurs with the amount of time spent writing and receiving SMS. Since we did not have a measure of the time spent in composing and reading SMS, we refrain from comparing past results [5] with our study that found that low-openness was a factor contributing to high SMS usage. Further, our study shows that introversion correlates with higher Internet usage and the number of SMS sent. Similar results have been obtained in previous studies $[13,2]$.

Apart from usage cues pertaining to calls and SMS, other features such as applications usage and BT scans have not been explored in the past. Hence these results cannot be compared with past work. Application us- 
Table 6: Results of multiple regression with feature as dependent variable and the five traits as independent variables.

\begin{tabular}{|c|c|c|c|c|c|}
\hline Feature & $\mathbf{R}^{2}$ & $\mathbf{F}$ & Feature & $\mathbf{R}^{2}$ & $\mathbf{F}$ \\
\hline Uses of Office & 0.12 & $7.07^{*}$ & Avg. word length (Sent Items) & 0.06 & $6.79^{*}$ \\
\hline Uses of Internet & 0.09 & $9.01^{*}$ & Median word length in (Sent Items) & 0.06 & $6.79^{*}$ \\
\hline Uses of Video/Audio/Music & 0.04 & $3.94^{*}$ & No. messages (Sent Items) & 0.07 & $7.84^{*}$ \\
\hline Uses of Maps & 0.05 & 2.21 & Outgoing calls & 0.01 & 0.6 \\
\hline Uses of Mail & 0.1 & $3.13^{\dagger}$ & Avg. duration of outgoing $(\mathrm{O})$ calls & 0 & 0.26 \\
\hline Uses of Youtube & 0.58 & $9.84^{*}$ & Total duration of outgoing calls & 0 & 0.27 \\
\hline Uses of Calendar & 0.06 & $4.81^{*}$ & Incoming calls & 0.07 & $8.29^{*}$ \\
\hline Uses of Camera & 0.04 & 2.24 & Avg. duration of incoming (I) calls & 0.06 & $6.25^{*}$ \\
\hline Uses of Chat & 0.41 & 0.96 & Total duration of incoming calls & 0.07 & 8.22 * \\
\hline Uses of SMS & 0.07 & $7.77^{*}$ & Unique contacts called & 0.01 & 0.65 \\
\hline Uses of Games & 0.63 & 3.01 & Unique contacts that called & 0.05 & $5.84^{*}$ \\
\hline Number of unique BT IDs & 0.03 & $3.22 *$ & Total I+O Calls & 0.01 & 1.05 \\
\hline Times most common BT ID is seen & 0.03 & $3.31^{*}$ & Avg. duration of $\mathrm{I}+\mathrm{O}$ Calls & 0.01 & 1.1 \\
\hline BTIDs accounting for $50 \%$ of IDs seen & 0.03 & $3.72 *$ & Total duration of I+O Calls & 0.01 & 1.22 \\
\hline BT IDs seen more than 4 times & 0.04 & $4.66^{*}$ & Unique contacts communicated with & 0.01 & 1.22 \\
\hline BT IDs seen more than 9 times & 0.03 & $3.84^{*}$ & Missed calls & 0.04 & $4.12^{*}$ \\
\hline BT IDs seen more than 19 times & 0.03 & $3.21^{*}$ & Unique contacts associated with & 0.02 & 1.64 \\
\hline Max. time for which a BT ID is seen & 0.03 & $3.04^{\dagger}$ & missed calls & & \\
\hline Avg. word length (Inbox) & 0.02 & 1.82 & SMS received & 0.03 & $3.02^{\dagger}$ \\
\hline Median word length in (Inbox) & 0.02 & 1.82 & Unique contacts SMS received from & 0.02 & 1.79 \\
\hline \multirow[t]{2}{*}{ No. messages (Inbox) } & 0.03 & $3.78^{*}$ & SMS sent & 0.06 & $6.92^{*}$ \\
\hline & & & Unique contacts SMS sent to & 0.03 & $2.85^{\dagger}$ \\
\hline
\end{tabular}

${ }^{\dagger} p<0.05 * p<0.01$

age can be an important source of information, given the emergence of the "app" culture in many popular smartphones. Our study is based on apps on Nokia N95 phones. Extension of this work to other brands such as iPhone and Android requires further study.

\subsection{User Classification}

In order to put our analysis into a machine learning framework, we defined a binary classification task for each of the Big-5 traits, using the median value of the traits, in the given sample, to split the data into two classes. This was done with an intention to detect, for example, the more extraverted and more introverted users and so on, in the given sample. All user-months having at least 7 -days of activity were used. We used a C4.5 classifier, which uses decision-trees for classification [21] and an SVM classifier with RBF kernel implemented through the Shogun Toolbox [23]. Before training the classifiers, only cues (described in Section 3) that exhibited correlations that were significant to a degree of $p<0.1$, and a maximum of 20 in number, from each source were pooled as input features for the classification task. Before using the SVM, all the variables were de-correlated and dimensions contributing to $99.99 \%$ of the variance were kept. Variables were further normalized, using the training data, so that they were bounded within $[-1,1]$. Leave-one-out cross-validation was done to obtain the average performance. The baseline was chosen as a classifier that chooses the majority class all the time. The average
Table 7: Classification results in \% accuracies across all folds of leave-one-out cross-validation

\begin{tabular}{lccl}
\hline trait & C4.5 & SVM & Baseline \\
\hline Extraversion & 61.1 & $75.9^{*}$ & 58.6 \\
Agreeableness & $68.5^{*}$ & $69.6^{*}$ & 58.4 \\
Conscientiousness & $68.1^{\dagger}$ & $74.4^{*}$ & 62.2 \\
Emotional Stability & $59.8^{*}$ & $71.5^{*}$ & 52.2 \\
Openness to Experience & $66.4^{*}$ & $69.3^{*}$ & 59.0 \\
\hline$\dagger p<0.1^{*} p<0.05$ & &
\end{tabular}

${ }^{\dagger} p<0.1{ }^{*} p<0.05$

accuracy across all folds is given in Table 7 . The results show that the users can be classified significantly above chance level for all traits, highlighting that aggregations of usage features from smartphone data can indeed be used to segment users, based on their personality traits. The traits for which accuracy was highest are extraversion and conscientiousness. Interestingly, other work on recognizing traits automatically has also found extraversion to be a feasible task [19].

\section{Conclusions}

In this paper, we have analyzed the relationship between smartphone usage and self-perceived personality. Applications usage, call and SMS logs contained several meaningful relationships to the Big-Five personality framework. Our study is based on a large-scale dataset of 8 months of real usage of smartphones by 83 people and personality surveys that are suitable for large mobile or online studies. 
Several results in our work concurred with the very few previous studies on survey-based self-perceived usage of phones and personality [5, 18]. Also, we found that Internet usage was correlated with introversion, which reinforces results observed in past work [13].

In contrast to previous work, our feature set was enriched with information automatically extracted from a variety of sources and collected from smartphones of a large number of users, using a non-intrusive software. Further, we were able to develop an automated system for classifying users based on the Big-Five personality traits. These classifiers performed above the level of chance. This is a promising result from the perspective of computational social science and its applications [14] and justifies further investigation into the discovery of user categories based on their personality.

In the future, we plan to study relationships between users and personality, by building social networks with the rich contextual information available in our data. Also, analyzing other modalities such as accelerometers and GPS logs remains a topic of further study.

\section{Acknowledgments}

This work was funded by the SNSF project Sensing and Analyzing Organizational Nonverbal Behavior (SONVB) and by Nokia Research Center (NRC) Lausanne. We thank Juha K. Laurila (NRC) and TrinhMinh-Tri Do (Idiap) for valuable discussions.

\section{References}

[1] I.c.t. statistics. http://www.itu.int/ITU-D/ict/ statistics/ict/index.html, Accessed 9 Feb 2011.

[2] Y. Amichai-Hamburger, G. Wainapel, and S. Fox. On the internet no one knows i'm an introvert: extroversion, neuroticism and internet interaction. CyberPsychology and Behaviour, 5:125-128, 2002.

[3] M. D. Back, J. M. Stopfer, S. Vazire, S. Gaddis, S. C. Schmukle, B. Egloff, and S. D. Gosling. Facebook profiles reflect actual personality, not self-idealization. Psychological Science, 21:372-374, 2010.

[4] W. P. Brinkman and N. Fine. Towards customized emotional design: an explorative study of user personality and user interface skin preferences. In Proc. Annual Conf. on European Association of Cognitive Ergonomics (EACE), 2005.

[5] S. Butt and J. G. Phillips. Personality and self reported mobile phone use. Computers in Human Behavior, 24:346 - 360, 2008.

[6] S. Counts and K. Stecher. Self-presentation of personality during online profile creation. In Proc. AAAI Conf. on Weblogs and Social Media (ICWSM), 2009.

[7] T. Do and D. Gatica-Perez. By their apps you shall understand them: mining large-scale patterns of mobile phone usage. In Proc. Int. Conf. on Mobile and Ubiquitous Multimedia (MUM), 2010.

[8] N. Eagle and A. Pentland. Eigenbehaviors: identifying structure in routine. Behavioral Ecology and Sociobiology, 63:1057-1066, 2009.
[9] K. Farrahi and D. Gatica-Perez. Probabilistic mining of socio-geographic routines from mobile phone data. IEEE Jrnl. of Selected Topics in Signal Processing, 4:746-755, 2010.

[10] S. D. Gosling, P. J. Rentfrow, and W. Swann. A very brief measure of the big-five personality domains. Jrnl. of Research in Personality, 37:504 - 528, 2003.

[11] A. Karsvall. Personality preferences in graphical interface design. In Proc. of the second Nordic conference on Human-computer interaction (NordiCHI), 2002.

[12] N. Kiukkonen, J. Blom, O. Dousse, D. Gatica-Perez, and J. Laurila. Towards rich mobile phone datasets: Lausanne data collection campaign. In Proc. ACM Int. Conf. on Pervasive Services (ICPS), 2010.

[13] R. Kraut, M. Patterson, V. Lundmark, S. Kiesler, T. Mukapadhyay, and W. Scherlis. Internet-paradox: a social technology that reduces social involvement and psychological well being? American Psychologist, 53:1017-1031, 1998.

[14] D. Lazer, A. Pentland, L. Adamic, S. Aral, A. Barbasi, D. Brewer, N. Christakis, N. Contractor, J. Fowler, M. Gutmann, T. Jebara, G. King, M. Macy, D. Roy, and M. V. Alstyne. Computational social science. Science, 323:721-723, 2009.

[15] F. Mairesse and M. Walker. Automatic recognition of personality in conversation. In Proc. Human Language Technology Conference of the NAACL, Companion Volume: Short Papers (NAACL-Short), 2006.

[16] F. Mairesse, M. Walker, M. R. Mehl, and R. K. Moore. Using linguistic cues for the automatic recognition of personality in conversation and text. Jrnl. of Artificial Intelligence Research, 30:457-500, 2007.

[17] R. R. McCrae and O. P. John. An introduction to the five-factor model and its applications. Jrnl. of Personality, 60:175-215, 1992.

[18] J. G. Phillips, S. Butt, and A. Blaszczynski. Personality and self-reported use of mobile phones for games. Cyberpsychology and Behavior, 9:753-758, 2006.

[19] F. Pianesi, N. Mana, A. Cappelletti, B. Lepri, and M. Zancanaro. Multimodal recognition of personality traits in social interactions. In Proc. Int. Conf. on Multimodal interfaces (ICMI), 2008.

[20] S. Poschl and N. Doring. Integration and Ubiquity. Towards a Philosophy of Telecommunications Convergence, chapter Personality and the Mobile Phone: Character-Based Differences of Usage and Attitudes towards Mobile Communication, pages 161-168. Vienna, Passagen Verlag, 2007.

[21] R. Quinlan. C4.5 classifier. http://www.rulequest. com/Personal/c4.5r8.tar.gz, Accessed Feb 2011.

[22] J. J. Romero. Top 11 technologies of the decade. IEEE Spectrum, pages 24-27, Feb. 2011.

[23] S. Sonnenburg, G. Raetsch, S. Henschel, C. Widmer J. Behr, A. Zien, F. D. Bona, A. Binder, C. Gehl, and V. Franc. The shogun machine learning toolbox. Jrnl. of Machine Learning Research, 11:1799-1802, 2010.

[24] K. Stecher and S. Counts. Spontaneous inference of personality traits and effects on memory for online profiles. In Proc. Int. AAAI Conference on Weblogs and Social Media (ICWSM), 2008.

[25] H. Verkasalo, C. Lpez-Nicols, F. J. Molina-Castillo, and H. Bouwman. Analysis of users and non-users of smartphone applications. Telematics and Informatics, $27: 242-255,2010$.

[26] T. Yeo. Modeling personality influences on youtube usage. In Proc. Int. AAAI Conference on Weblogs and Social Media (ICWSM), 2010. 